\title{
Malnutrition in HIV-Infected Children Is an Indicator of Severe Disease with an Impaired Response to Antiretroviral Therapy
}

\author{
Maximilian Muenchhoff, ${ }^{1,4, \star}$ Michael Healy, ${ }^{5,{ }^{\star}}$ Ravesh Singh,2 Julia Roider, ${ }^{1,2,6}$ Andreas Groll, \\ Chirjeev Kindra, Thobekile Sibaya, Angeline Moonsamy, Callum McGregor, ${ }^{1,2}$ Michelle Q. Phan, \\ Alejandro Palma, Henrik Kloverpris, ${ }^{6,9}$ Alasdair Leslie, ${ }^{6,10}$ Raziya Bobat, ${ }^{8,11}$ Philip LaRussa, ${ }^{12}$ \\ Thumbi Ndung'u, 2,6,10,13 Philip Goulder, ${ }^{1,2,14}$ Magdalena E. Sobieszczyk, and Mohendran Archary ${ }^{8,11}$
}

\begin{abstract}
This observational study aimed to describe immunopathogenesis and treatment outcomes in children with and without severe acute malnutrition (SAM) and HIV-infection. We studied markers of microbial translocation (16sDNA), intestinal damage (iFABP), monocyte activation (sCD14), T-cell activation (CD38, HLA-DR) and immune exhaustion (PD1) in $32 \mathrm{HIV}$-infected children with and $41 \mathrm{HIV}$-infected children without SAM prior to initiation of antiretroviral therapy (ART) and cross-sectionally compared these children to 15 HIV-uninfected children with and 19 HIV-uninfected children without SAM. We then prospectively measured these markers and correlated them to treatment outcomes in the HIV-infected children at 48 weeks following initiation of ART. Plasma levels of 16sDNA, iFABP and sCD14 were measured by quantitative real time PCR, ELISA and Luminex, respectively. T cell phenotype markers were measured by flow cytometry. Multiple regression analysis was performed using generalized linear models (GLMs) and the least absolute shrinkage and selection operator (LASSO) approach for variable selection. Microbial translocation, T cell activation and exhaustion were increased in HIV-uninfected children with SAM compared to HIV-uninfected children without SAM. In HIV-infected children microbial translocation, immune activation, and exhaustion was strongly increased but did not differ by SAM-status. SAM was associated with increased mortality rates early after ART initiation. Malnutrition, age, microbial translocation, monocyte, and CD8 T cell activation were independently associated with decreased rates of CD4\% immune recovery after 48 weeks of ART. SAM is associated with increased microbial translocation, immune activation, and immune exhaustion in HIV-uninfected children and with worse prognosis and impaired immune recovery in HIV-infected children on ART.
\end{abstract}

\footnotetext{
${ }^{1}$ Department of Paediatrics, University of Oxford, Peter Medawar Building for Pathogen Research, Oxford, United Kingdom.

${ }^{2}$ HIV Pathogenesis Programme, Doris Duke Medical Research Institute, Nelson R. Mandela School of Medicine, University of KwaZulu-Natal, Durban, South Africa.

${ }^{3}$ Department of Virology, Max von Pettenkofer-Institute, Ludwig-Maximilians-University Munich, Munich, Germany.

${ }^{4}$ German Center for Infection Research (DZIF), Munich, Germany.

${ }^{5}$ Division of Infectious Diseases, Department of Medicine, Columbia University, New York, New York.

${ }^{6}$ KwaZulu-Natal Research Institute for Tuberculosis and HIV (K-RITH), Nelson R Mandela School of Medicine, University of KwaZulu-Natal, Durban, South Africa.

${ }^{7}$ Department of Mathematics, Ludwig-Maximilians-University of Munich, Munich, Germany.

${ }^{8}$ King Edward VIII Hospital, Durban, South Africa.

${ }^{9}$ Department of Immunology and Microbiology, University of Copenhagen, Copenhagen, Denmark.

${ }^{10}$ The Ragon Institute of Massachusetts General Hospital, Massachusetts Institute of Technology and Harvard University, Cambridge, Massachusetts.

${ }^{11}$ Department of Paediatrics and Child Health, Nelson R. Mandela School of Medicine, University of KwaZulu-Natal, Durban, South Africa.

${ }^{12}$ Division of Infectious Diseases, Department of Pediatrics, Columbia University, New York, New York.

${ }^{13}$ Max Planck Institute for Infection Biology, Berlin, Germany.

${ }^{14}$ Department of Paediatric Infectious Diseases, Great Ormond St. Hospital for Children, London, United Kingdom.

*These authors contributed equally to this work.
}

(C) Maximilian Muenchhoff et al. 2018; Published by Mary Ann Liebert, Inc. This is an Open Access article distributed under the terms of the Creative Commons Attribution License, which permits unrestricted use, distribution, and reproduction in any medium, provided the original work is properly cited. 
Keywords: malnutrition, microbial translocation, immune activation, immune exhaustion, pediatric HIV, antiretroviral therapy

\section{Introduction}

$\mathbf{M}$ ALNUTRITION WAS ESTIMATED to be the underlying cause for $45 \%$ of deaths in children less than 5 years of age in 2011 globally. ${ }^{1}$ The prevalence of malnutrition in HIVinfected children is as high as $40 \%$, making clinical management of this vulnerable population even more challenging. 2,3 Co-occurrence of severe acute malnutrition (SAM) and HIV results in increased morbidity and mortality largely due to increased incidence and severity of concurrent infections. ${ }^{4-6}$ Immunological alterations such as systemic inflammation and impaired cellular immune responses have been attributed to malnutrition ${ }^{7}$ that could explain this increased susceptibility to infection especially when exacerbated by HIV infection, but the underlying mechanisms remain largely unresolved.

Malnutrition is characterized by functional and structural alterations in the intestinal mucosa associated with chronic intestinal inflammation. ${ }^{8}$ Direct effects of malnutrition ${ }^{9}$ and perturbations in the gut microbiome ${ }^{10}$ that are only partially restored after nutritional interventions ${ }^{11,12}$ have been proposed as a primary cause for persistent intestinal inflammation and epithelial damage. In HIV infection breakdown of the intestinal barrier, depletion of gut-resident $\mathrm{CD}^{+} \mathrm{T}$ cell populations and mucosal immune dysregulation results in microbial translocation that drives systemic immune activation. ${ }^{13}$ Chronic immune activation is a hallmark of disease progression in HIV-infected children and results in accelerated loss of CD4 T cells, immune dysregulation, and immune exhaustion. ${ }^{14}$

In HIV-infected children, it is not known whether SAM is associated with increased microbial translocation, immune activation, and immune exhaustion and whether this is modulated by ART. We sought to describe and compare levels of microbial translocation and immune activation and exhaustion in HIVuninfected and HIV-infected ART-naive children presenting with or without SAM and after 48 weeks of ART. We hypothesize that (i) malnutrition is associated with microbial translocation, immune activation, and immune exhaustion in HIV-infected and uninfected children; (ii) malnutrition, microbial translocation, and immune activation are associated with detrimental treatment outcome in HIV-infected children on ART. The current knowledge of microbial translocation and immunopathogenesis in the context of SAM and HIV is limited. Findings from this study may help to inform about the pathogenesis of malnutrition and HIV infection and guide additional interventions in the management of this critically ill population.

\section{Materials and Methods}

\section{Study subjects and procedures}

We studied four different groups of children aged 2 months to 12 years with the following inclusion criteria: (i) HIV+ SAM+: Presenting with SAM, confirmed HIV infection, ARTnaive; (ii) HIV+SAM-: No SAM, confirmed HIV infection, ART-naive; (iii) HIV-SAM+: Presence of SAM, confirmed negative HIV status; and (iv) HIV-SAM-: No SAM, confirmed negative HIV status, absence of severe acute or chronic medical disease. Exclusion criteria for all groups were lack of consent/assent or previous admission for SAM.
$\mathrm{HIV}+\mathrm{SAM}+(n=32)$ children were recruited as part of the recently described MATCH cohort ${ }^{15}$ (Clinical trial registry number: PACTR 21609001751384) from the pediatric ward of the King Edward VIII Hospital, Durban, South Africa, between July 2012 and March 2015. All other groups were recruited independent of the MATCH cohort between March 2013 and June 2014. HIV+SAM- children were recruited from the pediatric wards and outpatient clinic at the King Edward VIII Hospital $(n=20)$ and from the outpatient clinic at the Ithembalabantu Clinic, Umlazi, Durban, South Africa $(n=21)$. HIV-SAM+ $(n=15)$ children were recruited from the pediatric wards of the King Edward VIII Hospital. HIVuninfected siblings of HIV+SAM- children $(n=19)$ were recruited as HIV-SAM- group. Numbers of subjects at each stage of the study are summarized in Supplementary Figure S1 (Supplementary Data are available online at www .liebertpub.com/aid). All study participants were black African children mainly of Zulu and Xhosa origin.

HIV status was diagnosed as per the South African National HIV Treatment Guidelines ${ }^{16}$ by two independent ELISA/ Western blot assays in children above 18 months of age and two DNA PCR assays (TaqMan HIV-1 Qualitative Test; Roche Molecular Systems, Inc.) in children under 18 months by the National Health Laboratory Service (NHLS), South Africa.

SAM was defined based on 2009 WHO criteria ${ }^{17}$ as presence of: (i) weight-for-height more than three SD below the mean, (ii) mid-upper arm circumference $<115 \mathrm{~mm}$, or (iii) the presence of bilateral lower extremity pitting edema of nutritional origin. The WHO Anthro software package 3.2.2 for SPSS was used to calculate anthropometric z-scores. All children with SAM were admitted and managed based on the WHO guidelines for the in-patient treatment of severely malnourished children with standardized refeeding guidelines. ${ }^{17}$ Children were only discharged once they had reached nutritional recovery as defined in these guidelines. All children with SAM received antibiotics (Penicillin and Gentamicin) and antihelminthics (albendazole). HIV+SAM+ children additionally received Cotrimoxazole prophylaxis. All HIV-infected children were initiated on ART according to the current South African guidelines ${ }^{16}$ using a combination of abacavir and lamivudine plus either efavirenz or ritonavir boosted lopinavir. HIV+SAM+ children were initiated on ART after a median time of 11 days following hospitalization. Baseline blood samples were taken on the same day before the first dose of ART was given. HIV-positive children with SAM were followed up at week 4, 8, 12, 24, and 48 and without SAM at week 12, 24, and 48 only. All children were screened for active tuberculosis disease and if indicated treated according to national guidelines.

All research blood samples were collected in Ethylenediaminetetraacetic acid tubes and processed within $6 \mathrm{~h}$ after venipuncture at the HIV Pathogenesis Programme (HPP), UKZN, Durban, South Africa. Plasma was separated by centrifugation and cryopreserved at $-80^{\circ} \mathrm{C}$. Peripheral blood mononuclear cells (PBMCs) were isolated by Ficoll density gradient centrifugation and used for flow cytometric analysis immediately.

Written informed consent was obtained from caregivers for all study participants and, in addition, assent to participate 
in the study was given directly by children in the appropriate age groups. The study was reviewed and approved by the University of KwaZulu-Natal Ethics Review Board, Columbia University Institutional Review Board, and the Oxford Research Ethics Committee.

\section{CD4 count and viral load measurements}

Viral load levels were determined by the NHLS using the COBAS AmpliPrep/COBAS TaqMan HIV-1 Test v2.0 by Roche. ${ }^{18} \mathrm{CD} 4^{+} \mathrm{T}$ cell counts and percentage were measured by the NHLS using flow cytometry.

\section{Quantification of plasma biomarkers}

Plasma levels of sCD14 were quantified using a Luminex Kit (R\&D systems, Minneapolis, MN) at a 100-fold dilution in duplicate as per manufacturer's recommendations on a calibrated Luminex 200 analyzer (Luminex Corporation, Austin, TX). Plasma concentrations of intestinal fatty acid-binding protein (iFABP) were quantified using an ELISA Kit (R\&D systems, Minneapolis, MN) in duplicate at a 1:10 dilution according to the manufacturer's instructions. Results were expressed in $\mathrm{pg} / \mathrm{ml}$.

\section{Real-time PCR quantitation of $16 S$ rDNA in plasma}

To minimize the risk of contamination, blood samples were obtained using standard aseptic techniques following cleansing of the skin with $2 \%$ chlorhexidine/alcohol solution. Subsequently, all samples were handled in laminar flow cabinets under sterile conditions. Total DNA was extracted from $200 \mu$ l cryopreserved plasma using the column-based QIAamp DNA Mini Kit (Qiagen). 16S rDNA was amplified using the following published primers ${ }^{19}: 5^{\prime}$ - AGAGTTTGATCCTGGCTCAG -3' (forward) and 5'- CTGCTGCCTCCCGTAGGAGT - 3' (reverse). Each PCR comprised $3.5 \mathrm{mmol} / \mu \mathrm{l} \mathrm{MgCl} 2,0.125 \mathrm{pmol} / \mu \mathrm{l}$ of each primer, $5 \mu \mathrm{l}$ of SYBR Green I Master Mix (2X) (Roche), $2 \mu \mathrm{l}$ of DNA, and water to $10 \mu \mathrm{l}$. Reactions were run in triplicate on a Roche LightCycler 480 version 1.5 as follows: 1 cycle at $95^{\circ} \mathrm{C}$ for $10 \mathrm{~min}$, followed by 45 cycles at $95^{\circ} \mathrm{C}$ for $30 \mathrm{~s}$, at $60^{\circ} \mathrm{C}$ for $30 \mathrm{~s}$, and at $72^{\circ} \mathrm{C}$ for $30 \mathrm{~s}$. A standard curve was created from serial dilutions of $\mathrm{DH} 5 \alpha$ bacterial DNA containing known copy numbers of the template calculated by molecular weight as described previously. ${ }^{20}$ Individual samples were then analyzed against the standard curve, and 16S rDNA copy numbers per microliter of plasma were calculated.

\section{Surface phenotypic staining and flow cytometry}

PBMCs were used for staining with fluorochrome-conjugated monoclonal antibodies against cell surface markers for $\mathrm{T}$ cell activation, exhaustion, and memory differentiation as described previously. ${ }^{21}$ Briefly, freshly isolated PBMCs were washed in phosphate buffered saline, adjusted to two million cells per stain, and incubated with titrated concentrations of the following antibodies in a staining volume of $50 \mu \mathrm{L}$ in PBS: 1:100 CD3/BV605 (SK7; BD Biosciences), 1:100 CD4/V450 (RPA-T4; BD Biosciences), 1:100 CD8/V500 (RPA-T8; BD Biosciences), 1:50 PD-1/APC (MIH4; eBioscience), 1:25 CCR7/PE (150503; R\&D Systems), 1:50 CD45RA/Alexa Fluor 700 (HI100; BioLegend), 1:25 HLA-DR/FITC (L243; BD Biosciences), and 1:25 CD38/PE-Cy7 (HIT2; BD Biosciences). After $20 \mathrm{~min}$ incubation at room temperature cells were washed twice in PBS and resuspended in PBS with 2\% paraformaldehyde. Near-infrared LIVE/DEAD marker (Invitrogen) was used to exclude nonviable cells. Fluorescence minus one staining was used to determine the cutoff for PD1 high cells. Data were acquired on a LSR-II driven by FACSDiva software (BD) and analyzed using FlowJo software v8.8.6 (TreeStar, Ashland, OR). All FACS analyses were performed using freshly isolated PBMCs at the HIV Pathogenesis Programme, UKZN, Durban, South Africa.

\section{Statistical analyses}

Due to missing samples and incomplete clinical records, the dataset was incomplete. All available data are presented indicating the sample size for each parameter and group. Baseline characteristics were compared between study groups using Kruskal-Wallis ANOVA with Dunn's correction for multiple comparisons. Spearman correlations were used to explore bivariate associations. Kaplan-Meier survival curves were compared by the log-rank (Mantel-Cox) test. Statistical significance of differences in the prevalence of tuberculosis and viral suppression between study groups was calculated using Chi-Square tests. These statistical analyses were performed using GraphPad Prism version 6.0c (GraphPad Software, Inc., La Jolla, CA).

Multiple regression analysis was used to assess associations between clinical and immunological covariates with CD4 T cell immune activation in a Gaussian generalized linear model (GLM) with log link function using the statistical software R (R Development Core Team, 2014) with the R-package glm.

To study the associations among the parameters in this study a correlation matrix was plotted applying the R-package corrplot based on Spearman rank correlations with ordering of variables following first principal component analysis.

For the analysis of associations between baseline predictors and treatment outcomes, a model with favorable properties in regard to model complexity and statistical inference is needed due to the relatively small sample size in relation to the number of regarded covariates. Moreover, because of a high degree of correlation between some of the covariates, the model should be able to handle a certain level of multicollinearity. We therefore used an unbiased approach for variable selection applying the least absolute shrinkage and selection operator (LASSO) principle on scaled covariates. ${ }^{22}$ Subsequently, on the set of covariates selected by LASSO an un-regularized conventional GLM was fitted to obtain un-shrinked coefficient estimates together with standard errors. For better comparison of coefficient estimates between the LASSO and GLM, all covariates have been scaled. Observations with missing data for any of the regarded covariates were ignored and excluded from this analysis. For the regression analysis of the binary outcome, "W48 viral suppression" a binomial GLM with logit link function was used. For the continuous outcome "CD4\% immune recovery" a Gaussian GLM with log link function was chosen. "Viral suppression" was defined as viral load levels below limits of detection at the 48-week study visit. "Immune recovery of CD4\%" was defined as the difference of CD4\% at the 48-week study visit and baseline. The R-packages $\mathrm{glm}$ and glmnet $^{23}$ were used for these computations.

\section{Sample size determination}

There are currently no data describing microbial translocation in severely malnourished children with HIV infection. However, based on a prior study of sCD14 reduction after ART initiation in HIV-infected well-nourished children 
living in the United States,${ }^{24}$ we calculated that 20 patients per group will be needed to achieve a power of $80 \%$ to detect a difference in plasma sCD14 of within 1 SD between groups with $\alpha=0.05$. Anticipating potential loss to follow-up, patients were overenrolled in the HIV-infected groups to ensure sufficient subjects for the longitudinal part of this study.

\section{Results}

\section{Baseline characteristics}

Clinical characteristics of each study groups are summarized in Table 1. Among HIV-infected children, those with SAM were younger than their counterparts without SAM reflecting accelerated disease progression in this critically ill population that results in earlier clinical presentation. Children with SAM, regardless of HIV status, were stunted with low height for age $\mathrm{z}$-scores (haz) and showed signs of mixed, that is, chronic and acute, malnutrition as expressed in low weight for age (waz) combined with low weight for height $\mathrm{z}$-scores (whz). HIV+ SAM- children were mildly stunted with low haz scores, but normal waz and whz scores. Prevalence of active tuberculosis (TB) disease was higher in malnourished children with 15 cases out of $32 \mathrm{HIV}+\mathrm{SAM}+$ children and only 5 out of $41 \mathrm{HIV}+$ SAM- children at baseline ( $p=.0003$, Chi-square test). TB therapy was initiated as per current South African guidelines.

Levels of microbial translocation, intestinal damage, and monocyte activation differ by HIV- and SAM status

Microbial translocation, as measured by levels of bacterial 16sDNA in plasma, was significantly increased in all groups of
HIV-infected and malnourished children compared to healthy controls (Fig. 1A). Children with SAM had higher plasma levels of iFABP, a marker of intestinal damage, compared to children without SAM, regardless of HIV status (Fig. 1B). Soluble CD14 (sCD14), a marker of monocyte activation and inflammation, differed by HIV- but not by SAM status (Fig. 1C).

\section{Malnutrition and HIV infection are associated with increased immune activation}

We investigated $\mathrm{T}$ cell activation by measuring CD38 and HLA-DR expression in $\mathrm{CD}^{+}$and $\mathrm{CD}^{+} \mathrm{T}$ cells. Levels of immune activation in the $\mathrm{CD} 4^{+}$and $\mathrm{CD} 8^{+} \mathrm{T}$ cell compartment were highest in the HIV-infected study groups, but also significantly elevated in HIV-uninfected children with SAM compared to healthy controls (Fig. 1D-F). In regression analysis SAM $(\beta$-coefficient $=0.48, p$-value $=.0083)$ and HIV infection $(\beta$-coefficient $=1.04, p$-value $<.0001)$ were independently associated with increased $\mathrm{CD} 4^{+} \mathrm{T}$ cell activation controlling for age, sex, and TB status (Supplementary Table S1). However, there was no significant difference in $\mathrm{CD}^{+}$or $\mathrm{CD} 8^{+} \mathrm{T}$ cell activation between HIV-infected children with or without SAM.

\section{PD1 expression is increased in malnourished and HIV-infected children}

We next hypothesized that higher levels of immune activation in malnourished and HIV-infected children would be associated with increased immune exhaustion and studied PD1 expression, a marker of immune exhaustion. ${ }^{25}$ PD1

Table 1. Clinical Characteristics of Study Groups Defined by Hiv Status and Severe Acute Malnutrition at Baseline

\begin{tabular}{|c|c|c|c|c|}
\hline \multicolumn{5}{|c|}{ Baseline characteristics } \\
\hline Characteristics & $\begin{array}{c}H I V+S A M+ \\
(\mathrm{n}=32)\end{array}$ & $\begin{array}{c}H I V+S A M- \\
(\mathrm{n}=41)\end{array}$ & $\begin{array}{l}H I V-S A M+ \\
\quad(\mathrm{n}=15)\end{array}$ & $\begin{array}{l}H I V-S A M- \\
\quad(\mathrm{n}=19)\end{array}$ \\
\hline Age (years) & $1.6(0.7-3.4)$ & $8.2(4.5-10.7)$ & $0.9(0.5-1.5)$ & $9.1(6.8-12.8)$ \\
\hline Male sex & $18(56 \%)$ & $26(63 \%)$ & $7(47 \%)$ & $9(47 \%)$ \\
\hline $\mathrm{CD}^{+}{ }^{+} \mathrm{T}$ cell count, cells $/ \mathrm{mm}^{3}$ & $541(149-1167)$ & $412(212-875)$ & N/A & N/A \\
\hline CD4\% & $14.9(8.5-20)$ & $18.0(9-24.5)$ & N/A & N/A \\
\hline HIV RNA level, copies/ml & $\begin{array}{c}1.2 \times 10^{6} \\
\left(5.9 \times 10^{5}-7.3 \times 10^{6}\right)\end{array}$ & $\begin{array}{c}1.4 \times 10^{5} \\
\left(3.3 \times 10^{4}-5.9 \times 10^{5}\right)\end{array}$ & N/A & N/A \\
\hline Active tuberculosis disease & $15(47 \%)$ & $5(12 \%)$ & 0 & 0 \\
\hline Diarrhea & $2(6 \%)$ & $2(5 \%)$ & $7(50 \%)$ & 0 \\
\hline $\begin{array}{l}\text { Lower respiratory tract } \\
\text { infection }\end{array}$ & $6(19 \%)$ & $3(7 \%)$ & 0 & 0 \\
\hline Oral thrush & $5(16 \%)$ & $2(5 \%)$ & 0 & 0 \\
\hline WHO stage IV & $32(100 \%)$ & $8(20 \%)$ & N/A & N/A \\
\hline Admitted to hospital & $32(100 \%)$ & $5(12 \%)$ & $15(100 \%)$ & 0 \\
\hline Height for age $\mathrm{z}$ score (haz) & $-3.3(-4.7$ to -2.4$)$ & $-2.0(-2.8$ to -1$)$ & $-2.1(-3.6$ to -1.2$)$ & $-0.2(-1.6$ to 1$)$ \\
\hline Weight for age $\mathrm{z}$ score (waz) & $-3.4(-4.5$ to -3$)$ & $-0.9(-1.9$ to -0.3$)$ & $-1.8(-4$ to -1.4$)$ & $0.0(-0.5$ to 0.9$)$ \\
\hline $\begin{array}{l}\text { Weight for height } \mathrm{z} \\
\text { score (whz) }\end{array}$ & $-1.9(-3.6$ to -0.6$)$ & $0.5(-0.5$ to 1.4$)$ & $-1.2(-2.4$ to 0$)$ & $0.9(0.2-2.3)$ \\
\hline $\begin{array}{l}\text { Weight for height } \mathrm{z} \\
\text { score }(\mathrm{whz})<3\end{array}$ & $11(34 \%)$ & 0 & $1(7 \%)$ & 0 \\
\hline $\begin{array}{l}\text { Mid-upper arm } \\
\text { circumference }<115 \mathrm{~mm}\end{array}$ & $18(56 \%)$ & 0 & $4(27 \%)$ & 0 \\
\hline $\begin{array}{l}\text { Bilateral lower extremity } \\
\text { pitting edema }\end{array}$ & $13(42 \%)$ & 0 & $12(80 \%)$ & 0 \\
\hline
\end{tabular}

Median values with interquartile range or number of study subjects with percentage are shown for each characteristic by study group. SAM, severe acute malnutrition; N/A, not applicable. 

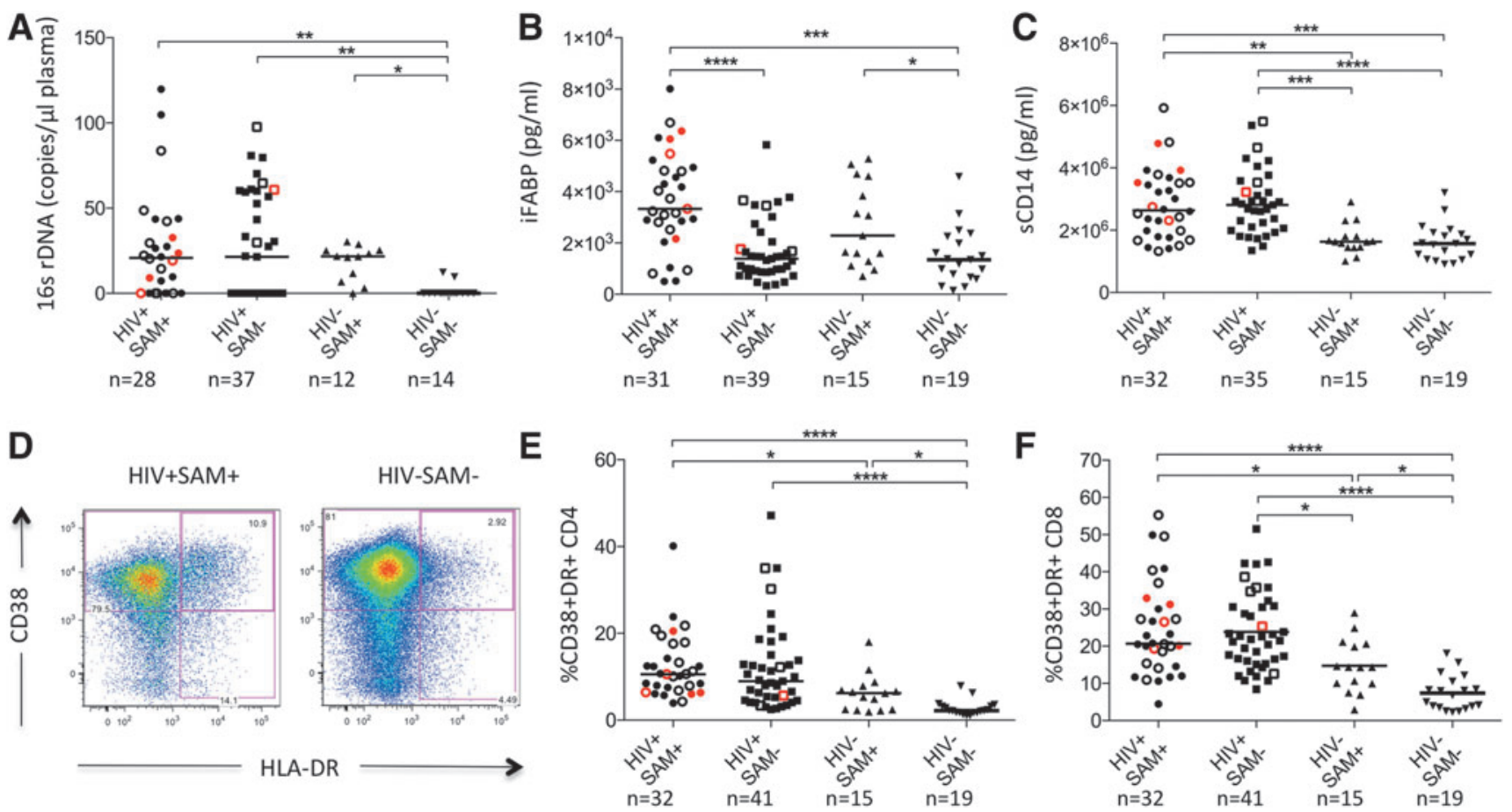

FIG. 1. Plasma biomarkers and immune activation by study group at baseline. (A-C) Plasma levels of 16sDNA (A), iFABP (B), and sCD14 (C) in children stratified by HIV status and presence of SAM at baseline. Open symbols indicate active tuberculosis disease; red symbols indicate children that passed away during the study period. Medians are shown as horizontal bars. (D) Representative $\mathrm{CD}^{+} \mathrm{T}$ cell immune activation FACS data from an HIV-positive child with severe acute malnutrition (HIV+SAM+, left) and a healthy control (HIV-SAM-, right). (E, F) Activation of CD4 ${ }^{+}$and CD8 ${ }^{+} \mathrm{T}^{-}$ cells in children stratified by HIV status and nutritional state. Kruskal-Wallis ANOVA with Dunn's correction for multiple comparisons was used to determine statistical significance $(* p<.05 ; * * p<.01 ; * * * p<.001 ; * * * * p<.0001)$. iFABP, intestinal fatty acid-binding protein; SAM, severe acute malnutrition.

surface expression was increased in $\mathrm{CD}^{+}$and $\mathrm{CD}^{+} \mathrm{T}$ cells in all malnourished and HIV-infected study groups (Fig. 2A-C).

Because PD1 expression is commonly higher in T cells with a more terminally differentiated memory phenotype, ${ }^{26}$ we also assessed PD1 expression on different memory subsets. $\mathrm{PD} 1$ expression was highest in the $\mathrm{CD} 4^{+}$and $\mathrm{CD}^{+} \mathrm{T}$ effector memory populations (Tem) and similarly increased in malnourished and HIV-infected children compared to healthy controls (Fig. 2D, E). Of note, while PD1 expression differed by SAM status in HIV-uninfected children, there was no significant difference by SAM status in HIV-infected children.

\section{Markers of microbial translocation, immune activation, and immune exhaustion correlate with each other and clinical markers of disease progression}

To better understand the nature of the associations between the immunological and clinical parameters in this study, we constructed a correlation matrix using data from all 107 participants at baseline. Variables were grouped based on principal component analysis, and results were visualized using the R package "corrplot" (Fig. 3). Parameters of immune activation, immune exhaustion, microbial translocation, and viral load are associated with disease cluster together based on strong positive correlations and are opposed to the second cluster of clinical parameters associated with health (i.e., high CD4\% and anthropometric measurements) based on negative correlations. A similar pattern was observed, including only HIV-positive children $(n=73)$ in the analysis (Supplementary Fig. S2).

\section{SAM is associated with higher mortality in HIV-infected children starting $A R T$}

All HIV-infected children were initiated on ART according to the current South African guidelines. During the 48week follow-up period, 5 of 32 children in the HIV+SAM+ group and 1 of 41 children in the HIV+SAM-group were deceased (Fig. 4A). The median time from enrollment to death was 31 days (range 9-131 days). Causes of death in the $\mathrm{HIV}+\mathrm{SAM}+$ children were bacterial infections with sepsis (3), pneumonia (1), gastroenteritis (1), and TB-meningitis in the HIV+SAM- child. The higher mortality rate of children with SAM was statistically significant ( $p=.047$, log-rank (MantelCox) test) and independent of active TB disease. Excluding all TB cases from the analysis, 3 out of 17 children in the $\mathrm{HIV}+\mathrm{SAM}+$ group died compared to 0 out of 36 children in the HIV+SAM- group ( $p=.0171$, log-rank (Mantel-Cox) test) (Fig. 4B). Four HIV+SAM- children (9.8\%) and $1 \mathrm{HIV+}$ SAM+ child $(3.1 \%)$ were lost to follow-up.

\section{Malnutrition, microbial translocation, and immune activation are associated with detrimental treatment outcomes in children initiating $A R T$}

For the $48 \mathrm{HIV}$-infected children who completed the follow-up period with available data for all parameters at 

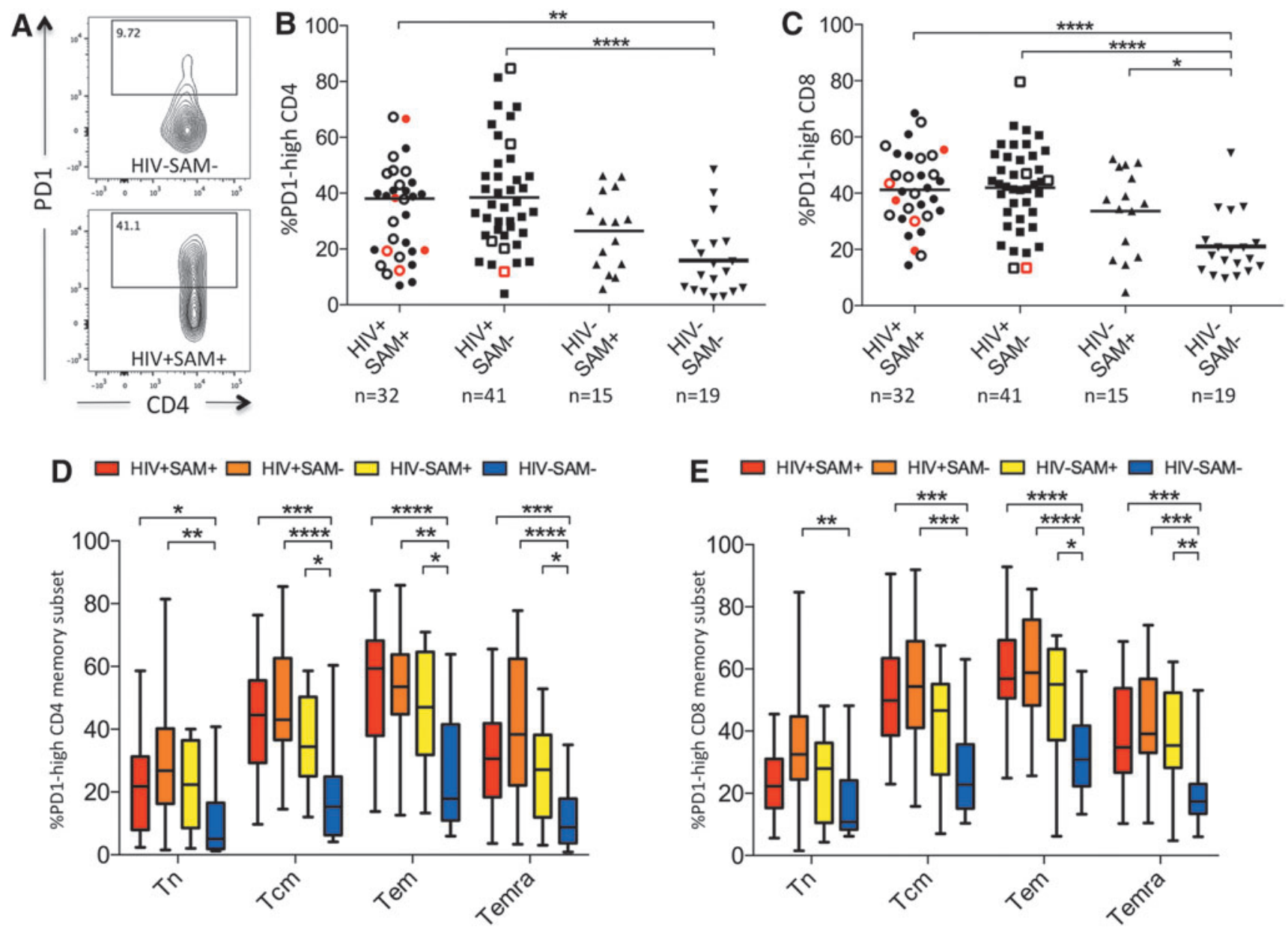

FIG. 2. PD1 expression by study group. (A) $\mathrm{CD}^{+} \mathrm{T}$ cell PD1 FACS data showing an FMO control (top) and a representative HIV+SAM+ sample (bottom). (B, C) PD1 expression in $\mathrm{CD}^{+}{ }^{+}$and $\mathrm{CD} 8^{+} \mathrm{T}$ cells by study group. Open symbols indicate active TB disease; red symbols indicate children that passed away during the study period. (D, E) PD1 expression on CD4 and CD8 $\mathrm{T}$ cell memory subsets defined by CD45RA and CCR7 expression (Tn: $\mathrm{CD}^{4} \mathrm{RA}^{+} \mathrm{CCR} 7^{+}$, Tcm: CD45RA ${ }^{-} \mathrm{CCR}^{+}$, Tem: CD45RA ${ }^{-} \mathrm{CCR}^{-}$, Temra: CD45RA ${ }^{+} \mathrm{CCR}^{-}$). Kruskal-Wallis ANOVA with Dunn's correction for multiple comparisons was used to determine statistical significance $(* p<.05 ; * * p<.01 ; * * * p<.001 ; * * * *<.0001)$.

baseline, we first assessed which variables were associated with viral suppression at week 48 . Because of the fairly large number of covariates compared to the relatively low number of cases studied here, we used the LASSO approach for variable selection. We then included these selected predictor variables in a GLM for confirmation. Active TB disease and high levels of sCD14 and 16sDNA at baseline were independently associated negatively with viral suppression at W48 (Table 2). Of note, there was no statistically significant difference for the proportion of children with viral suppression at week 48 between the two groups (11 of $18 \mathrm{HIV}+\mathrm{SAM}+$ children and 21 of $30 \mathrm{HIV}+$ SAM- children; $p=.53$, Chi-square test).

Subsequently we investigated the relationship of the clinical and immunological parameters at baseline with the rate of immune reconstitution as measured by recovery of CD4\% in children with viral suppression at the week 48 study visit. We in this study defined the rate of CD4\% recovery, that is, the difference of CD4\% between the week 48 study visit and baseline, as the dependent variable. For this analysis we only included the 32 children with viral suppression at the week 48 visit and with complete data for all parameters at baseline. Out of the set of 14 predictor variables, 8 variables were selected by the LASSO approach that was included in our final GLM. SAM, sCD14, 16sDNA, CD4\%, and $\mathrm{CD}^{+} \mathrm{T}$ cell activation at baseline were associated with lower rates of immune recovery at a statistically significant level in the confirmatory GLM (Table 2). These results demonstrate an association of SAM, increased microbial translocation, and systemic immune activation with impaired CD4\% recovery in children initiated on ART.

\section{Discussion}

While microbial translocation, immune activation, and immune exhaustion have been identified as key drivers in the pathogenesis of HIV disease, their role in malnutrition remains unclear. HIV-infected children with SAM likely represent a distinct clinical entity with a severe disease phenotype ${ }^{27}$ given that these children tend to present at a young age with increased comorbidities and high mortality. In this study, we show that SAM is associated with increased microbial translocation, immune activation, and immune exhaustion in HIVuninfected children and with an impaired response to ART in HIV-infected children. 

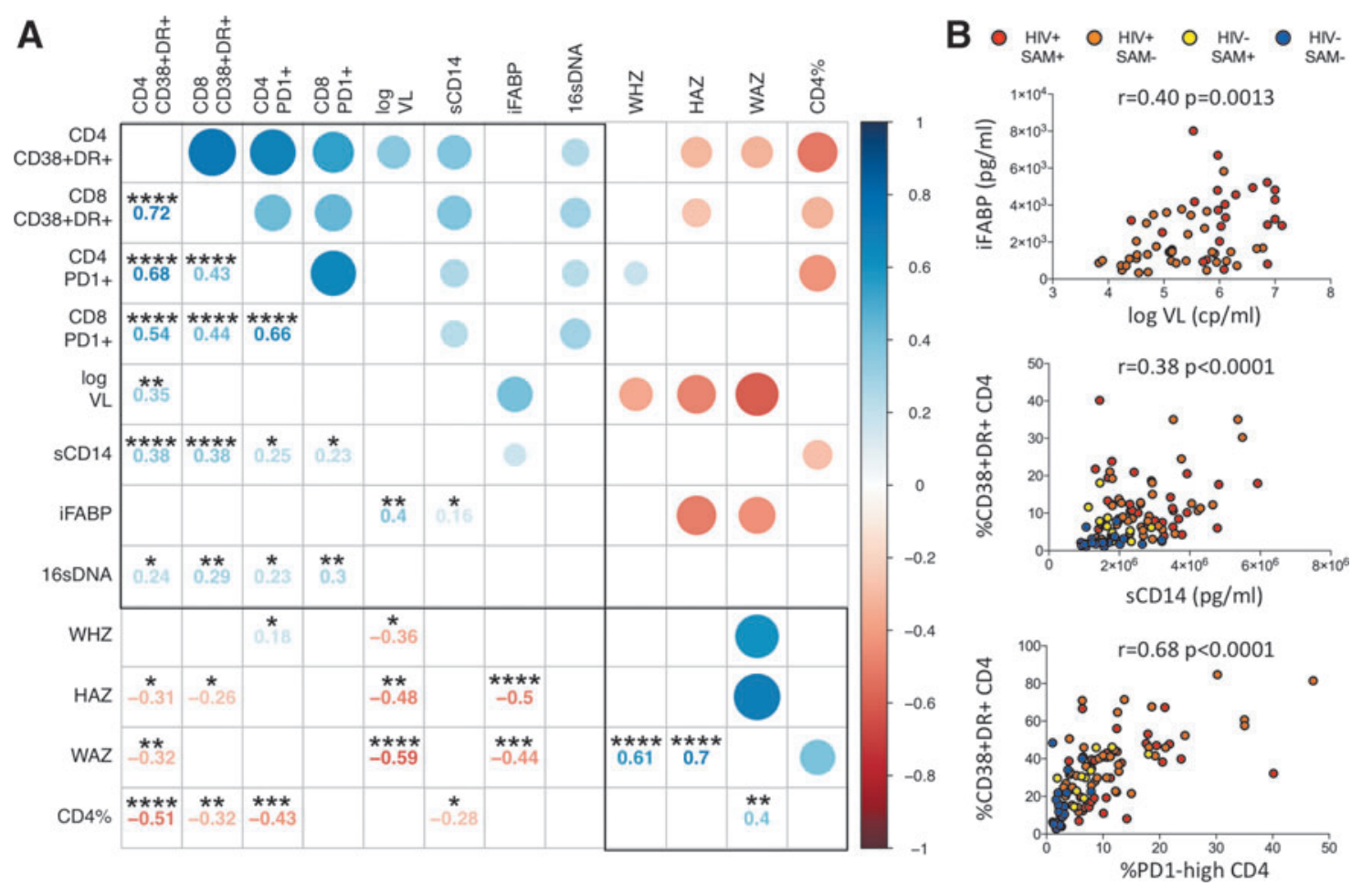

FIG. 3. Associations between plasma biomarkers and immunological and clinical variables. (A) Correlation matrix with available data from all participants at baseline $(n=107)$. Positive correlations are indicated in blue, and inverse correlations are indicated in red. Darker color shades indicate higher $r$-values. In the lower panel $r$ - and $p$-values are indicated based on Spearman's rank correlation tests $(* p<.05$; ** $p<.01$; *** $p<.001$; **** $p<.0001)$. Clustering of variables is based on principal component analysis using the $\mathrm{R}$ package corrplot and reveals two well-differentiated groups of parameters, one associated with disease (upper left quadrant) and the other associated with health (lower right quadrant). (B) Selected bivariate associations with Spearman rank correlation tests.

In our study, microbial translocation was not only increased in HIV-infected children as previously reported ${ }^{28,29}$ but also in HIV-uninfected malnourished children. Childhood malnutrition leads to alterations of the intestinal microbiome, chronic gut inflammation, and structural changes in the epithelium resulting in enteropathy with loss of intestinal barrier functions. ${ }^{7}$ We in this study show that plasma levels of iFABP, which is released by dying epithelial cells and used as a plasma marker of intestinal damage, ${ }^{30}$ were elevated in malnourished children independent of HIV status indicating structural intestinal damage that predisposes to microbial translocation.

Translocation of luminal bacterial products into the systemic circulation triggers systemic immune activation. ${ }^{13} \mathrm{In}$ pediatric HIV infection, chronic T cell activation is a key component of systemic immune activation and plays a central role in HIV pathogenesis. ${ }^{14,21}$ Consistent with previous reports ${ }^{28}$ we observed increased levels of T cell activation in HIV-infected children that correlated with markers of disease progression, microbial translocation, and monocyte activation. Earlier studies of malnourished children without apparent infections reported activation of the innate immune system with elevated leukocyte counts and increased acute phase responses. ${ }^{7} \mathrm{We}$ in this study show that $\mathrm{T}$ cell immune activation is also increased in HIV-uninfected malnourished children and confirmed an independent effect of SAM on $\mathrm{CD}^{+} \mathrm{T}$ cell activation controlling for HIV infection, age, sex, and TB status in a multivariate analysis. These data show that increased $\mathrm{T}$ cell immune activation forms part of the complex immunological alterations in malnutrition.
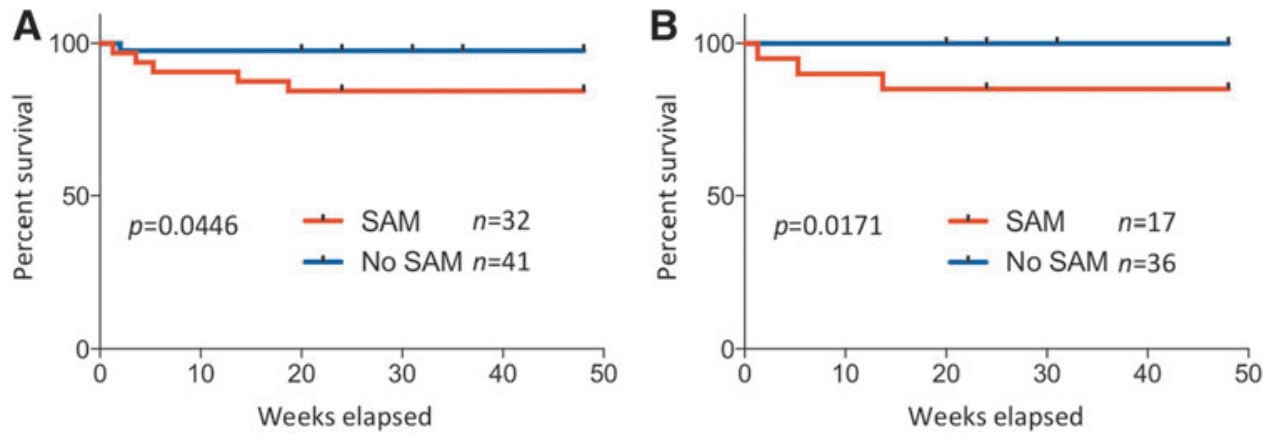

FIG. 4. Increased mortality in malnourished HIV-infected children on ART. (A) KaplanMeier curves comparing survival between all HIV-infected children with and without SAM. (B) Kaplan-Meier survival curves excluding all TB cases. Log-rank (Mantel-Cox) tests were applied for statistical comparison between the groups. 
Table 2. Association of Clinical and Immunological Predictors with Treatment Outcomes

Predictors associated with HIV treatment outcomes

\begin{tabular}{|c|c|c|c|c|c|c|c|}
\hline \multirow[b]{3}{*}{ Co-variable } & \multicolumn{3}{|c|}{ W48 viral suppression } & \multirow[b]{3}{*}{ Co-variable } & \multicolumn{3}{|c|}{ CD4\% immune recovery } \\
\hline & \multirow{2}{*}{$\frac{\text { LASSO }}{\beta \text {-coefficient }}$} & \multicolumn{2}{|c|}{$G L M$} & & \multirow{2}{*}{$\frac{L A S S O}{\beta \text {-coefficient }}$} & \multicolumn{2}{|c|}{$G L M$} \\
\hline & & $\beta$-coefficient & $\mathrm{p}$-value & & & $\beta$-coefficient & $\mathrm{p}$-value \\
\hline Active TB & -0.67 & -1.30 & 0.005 & sCD14 & -1.38 & -3.10 & 0.026 \\
\hline sCD14 & -0.74 & -1.47 & 0.006 & 16sDNA & -3.26 & -4.32 & 0.002 \\
\hline 16 sDNA & -0.50 & -1.37 & 0.025 & SAM & -0.99 & -4.07 & 0.019 \\
\hline SAM & 0 & - & - & Age & -1.84 & -3.43 & 0.037 \\
\hline Age & 0 & - & - & CD8 activation & -2.74 & -4.16 & 0.012 \\
\hline CD8 activation & 0 & - & - & CD $4 \%$ & -5.48 & -7.17 & $<0.0001$ \\
\hline CD $4 \%$ & 0 & - & - & iFABP & 0.49 & 0.7435 & 0.61 \\
\hline iFABP & 0 & - & - & CD4 activation & 0.38 & 1.63 & 0.30 \\
\hline CD4 activation & 0 & - & - & Active TB & 0 & - & - \\
\hline Viral load & 0 & - & - & Viral load & 0 & - & - \\
\hline Sex (male) & 0 & - & - & Sex (male) & 0 & - & - \\
\hline CD4 count & 0 & - & - & CD4 count & 0 & - & - \\
\hline CD4 PD1 & 0 & - & - & CD4 PD1 & 0 & - & - \\
\hline CD8 PD1 & 0 & - & - & CD8 PD1 & 0 & - & - \\
\hline CD4 $\mathrm{T}$ naive & 0 & - & - & $\mathrm{CD} 4 \mathrm{~T}$ naive & 0 & - & - \\
\hline
\end{tabular}

In the left panel, results are shown for analysis of the clinical outcome "W48 viral suppression" (i.e., viral load below detectable limits at the 48-week study visit) for all HIV-infected children with complete follow-up and available data for all parameters at baseline ( $n=48)$. LASSO selected three predictor variables, "active TB (tuberculosis) disease", "sCD14," and 16sDNA at baseline, to be negatively associated with successful viral suppression out of a set of 14 predictor variables. These three predictor variables were included in a generalized linear model (GLM) for confirmation. In the right panel, results are shown for analysis of recovery of CD4\% from baseline at week 48 , that is, the difference between CD4\% at the week 48 study visit and baseline. For this analysis, only children with viral suppression at the W48 study visit were included ( $n=32)$. LASSO selected eight predictors of which "sCD14," "16sDNA," "SAM" (severe acute malnutrition), "Age," "CD8 T cell activation," and "CD4\% at baseline" could be confirmed in a generalized linear model to be negatively associated with immune recovery at a statistically significant level. For better comparison of beta-coefficients, scaled data were used for all models.

However, we observed similar levels of immune activation in HIV-infected children with SAM compared to HIV-infected children with normal nutritional status indicating no further increase in immune activation due to malnutrition in HIV infection. Although we observed higher levels of iFABP in malnourished children regardless of HIV status indicative of more intestinal damage in these groups, this did not seem to result in higher levels of microbial translocation or immune activation in the HIV+SAM+ group possibly indicating that the high levels of immune activation and inflammation in HIV-infected children are not significantly modulated further by malnutrition.

Studies of lymphocyte function in malnourished children consistently showed deficits in cellular immunity such as reduced proliferative responses to PHA stimulation ${ }^{31}$ and impaired cytokine production upon in vitro stimulation. ${ }^{32-35}$ These functional deficits resemble the effects of immune exhaustion on $\mathrm{T}$ cell function as previously described in studies of chronic viral infections (LCMV, HIV) and cancer. ${ }^{36}$ We therefore hypothesized that the alterations in $\mathrm{T}$ cell function observed in malnourished children are associated with immune exhaustion and measured expression levels of PD1, a marker of immune exhaustion. ${ }^{25,37}$ PD1 expression on $\mathrm{T}$ cells was increased in malnourished HIV-uninfected children to levels similar to HIV-infected children. PD1 levels strongly correlated with $\mathrm{T}$ cell activation at baseline suggesting that immune exhaustion is driven by immune activation as previously hypothesized. ${ }^{38,39}$ Of note, PD1 expression did not differ by SAM status in HIV-infected children analogous to our observations for immune activation.
In the prospective part of this study we assessed the potential role of malnutrition, microbial translocation, and immune activation to predict treatment outcomes in HIV-positive children initiated on ART. Consistent with previous reports, ${ }^{4,5}$ SAM at baseline was highly predictive of all-cause mortality in children on ART. Monocyte activation, microbial translocation, and active TB disease were independently correlated inversely with viral suppression at 48 weeks of ART. Monocyte activation, microbial translocation, malnutrition, age, and $\mathrm{CD}^{+} \mathrm{T}$ cell activation at baseline were independently associated with impaired immune recovery. Of note, while our analysis did show a negative association between SAM and immune recovery, other markers such as CD4\%, sCD14, and CD8 T cell activation at baseline seemed to be of more predictive value for immune recovery. These findings are consistent with previous studies in HIV-infected adults that found high levels of PD-1 expression, $^{40-42} \mathrm{~T}$ cell activation ${ }^{43}$ and microbial translocation $^{44,45}$ to be associated with poor immune recovery.

Our study has a number of important limitations. First, there are profound differences between the study groups, including younger age and increased comorbidities in the children with SAM reflecting the severe disease phenotype in this critically ill population. The observed differences between the groups should therefore not solely be attributed to malnutrition per se, but malnutrition should rather be regarded as an important indicator of severe illness that is associated with profound immunological alterations. Second, in the prospective part of the study the number of participants with complete available data was relatively low and hence we only followed an exploratory hypothesis building approach in 
our analysis. These results should be validated in larger cohorts of malnourished HIV-infected children. Although we did not detect statistically significant differences between the sexes in our analyses likely due to the relatively low number of cases, future studies on microbial translocationespecially in the context of HIV-should take sex into account since sexual dimorphisms have previously been reported for both conditions. ${ }^{4,46,47}$ Finally, due to the observational character of this study we can only describe associations.

In summary, we demonstrate that SAM is an indicator of severe disease that is associated with worse prognosis and impaired immune recovery in HIV-infected children on ART prompting extensive medical attention for this population. Immune activation and microbial translocation are associated with impaired immune recovery in HIV-infected children initiated on ART and represent viable targets for additional interventions.

\section{Acknowledgments}

The authors thank the volunteers, patients, their families, and staff of the Pediatrics Department at King Edward VIII Hospital and the Ithembalabantu Clinic in Umlazi.

This work was supported by the Doris Duke Charitable Foundation International Research Fellowship; the Infectious Diseases Society of America (IDSA) Medical Scholars Program; the American Medical Association (AMA) Seed Grant; the International Family AIDS Program of Columbia University; and the Wellcome Trust [WT104748MA to P.G.].

The information in this article has not been presented at scientific meetings previously.

\section{Author Disclosure Statement}

No competing financial interests exist.

\section{References}

1. Black RE, Victora CG, Walker SP, et al.: Maternal and child undernutrition and overweight in low-income and middle-income countries. Lancet 2013;382:427-451.

2. Jesson J, Masson D, Adonon A, et al.: Prevalence of malnutrition among HIV-infected children in Central and West-African HIV-care programmes supported by the Growing Up Programme in 2011: A cross-sectional study. BMC Infect Dis 2015;15:216.

3. Anabwani G, Navario P: Nutrition and HIV/AIDS in subSaharan Africa: An overview. Nutrition 2005;21:96-99.

4. Munthali T, Jacobs C, Sitali L, Dambe R, Michelo C: Mortality and morbidity patterns in under-five children with severe acute malnutrition (SAM) in Zambia: A fiveyear retrospective review of hospital-based records (20092013). Arch Public Health 2015;73:23.

5. Preidis GA, McCollum ED, Mwansambo C, Kazembe PN, Schutze GE, Kline MW: Pneumonia and malnutrition are highly predictive of mortality among African children hospitalized with human immunodeficiency virus infection or exposure in the era of antiretroviral therapy. J Pediatr 2011;159:484-489.

6. Miller TL: Nutritional aspects of HIV-infected children receiving highly active antiretroviral therapy. AIDS 2003; 17 Suppl 1:S130-S140.
7. Rytter MJ, Kolte L, Briend A, Friis H, Christensen VB: The immune system in children with malnutrition-a systematic review. PLoS One 2014;9:e105017.

8. Campbell DI, Murch SH, Elia M, et al:: Chronic T cellmediated enteropathy in rural west African children: Relationship with nutritional status and small bowel function. Pediatr Res 2003;54:306-311.

9. Hashimoto T, Perlot T, Rehman A, et al.: ACE2 links amino acid malnutrition to microbial ecology and intestinal inflammation. Nature 2012;487:477-481.

10. Monira S, Nakamura S, Gotoh K, et al.: Gut microbiota of healthy and malnourished children in bangladesh. Front Microbiol 2011;2:228.

11. Smith MI, Yatsunenko T, Manary MJ, et al:: Gut microbiomes of Malawian twin pairs discordant for kwashiorkor. Science 2013;339:548-554.

12. Subramanian S, Huq S, Yatsunenko T, et al.: Persistent gut microbiota immaturity in malnourished Bangladeshi children. Nature 2014;510:417-421.

13. Brenchley JM, Price DA, Schacker TW, et al:: Microbial translocation is a cause of systemic immune activation in chronic HIV infection. Nat Med 2006;12:1365-1371.

14. Roider JM, Muenchhoff M, Goulder PJ: Immune activation and paediatric HIV-1 disease outcome. Curr Opin HIV AIDS 2016;11:146-155.

15. Archary M: HIV-infected children with severe acute malnutrition: Early vs delayed ART initiation. Available at www .croiconference.org/sessions/hiv-infected-children-severeacute-malnutrition-early-vs-delayed-art-initiation, accessed February 24, 2017.

16. Department of Health RoSA: The South African Antiretroviral Treatment Guidelines. Available at www.kznhealth .gov.za/medicine/2013_art_guidelines.pdf, accessed August 25,2016

17. WHO: Guideline: Updates on the Management of Severe Acute Malnutrition in Infants and Children. Available at http://apps.who.int/iris/bitstream/10665/95584/1/9789241 506328_eng.pdf, accessed August 25, 2016.

18. Pas S, Rossen JW, Schoener D, et al.: Performance evaluation of the new Roche Cobas AmpliPrep/Cobas TaqMan HIV-1 test version 2.0 for quantification of human immunodeficiency virus type 1 RNA. J Clin Microbiol 2010;48: 1195-1200.

19. Sandler NG, Wand H, Roque A, et al:: Plasma levels of soluble CD14 independently predict mortality in HIV infection. J Infect Dis 2011;203:780-790.

20. Simon TD, Van Yserloo B, Nelson K, et al.: Use of quantitative $16 \mathrm{~S}$ rRNA PCR to determine bacterial load does not augment conventional cerebrospinal fluid (CSF) cultures among children undergoing treatment for CSF shunt infection. Diagn Microbiol Infect Dis 2014;78:188195.

21. Muenchhoff M, Adland E, Karimanzira O, et al.: Nonprogressing HIV-infected children share fundamental immunological features of nonpathogenic SIV infection. Sci Transl Med 2016;8:358ra125.

22. Tibshirani R: Regression shrinkage and selection via the lasso. J R Stat Soc B 1996;58:267-288.

23. Friedman J, Hastie T, Tibshirani R: Regularization paths for generalized linear models via coordinate descent. J Stat Softw 2010;33:1-22.

24. Wallet MA, Rodriguez CA, Yin L, et al:: Microbial translocation induces persistent macrophage activation 
unrelated to HIV-1 levels or T-cell activation following therapy. AIDS 2010;24:1281-1290.

25. Day CL, Kaufmann DE, Kiepiela P, et al.: PD-1 expression on HIV-specific T cells is associated with T-cell exhaustion and disease progression. Nature 2006;443:350-354.

26. Yamamoto T, Price DA, Casazza JP, et al.: Surface expression patterns of negative regulatory molecules identify determinants of virus-specific CD8+ T-cell exhaustion in HIV infection. Blood 2011;117:4805-4815.

27. Heikens GT, Bunn J, Amadi B, et al.: Case management of HIV-infected severely malnourished children: Challenges in the area of highest prevalence. Lancet 2008;371:13051307.

28. Pilakka-Kanthikeel S, Kris A, Selvaraj A, Swaminathan S, Pahwa S: Immune activation is associated with increased gut microbial translocation in treatment-naive, HIVinfected children in a resource-limited setting. J Acquir Immune Defic Syndr 2014;66:16-24.

29. Fitzgerald F, Harris K, Doyle R, Alber D, Klein N: Short communication: Evidence that microbial translocation occurs in HIV-infected children in the United Kingdom. AIDS Res Hum Retroviruses 2013;29:1589-1593.

30. Pelsers MM, Namiot Z, Kisielewski W, et al.: Intestinaltype and liver-type fatty acid-binding protein in the intestine. Tissue distribution and clinical utility. Clin Biochem 2003;36:529-535.

31. Ortiz R, Campos C, Gomez JL, Espinoza M, RamosMotilla M, Betancourt M: Effect of renutrition on the proliferation kinetics of PHA stimulated lymphocytes from malnourished children. Mutat Res 1995;334:235-241.

32. Rodriguez L, Gonzalez C, Flores L, Jimenez-Zamudio L, Graniel J, Ortiz R: Assessment by flow cytometry of cytokine production in malnourished children. Clin Diagn Lab Immunol 2005;12:502-507.

33. Gerriets VA, MacIver NJ: Role of T cells in malnutrition and obesity. Front Immunol 2014;5:379.

34. Gonzalez-Martinez H, Rodriguez L, Najera O, et al.: Expression of cytokine mRNA in lymphocytes of malnourished children. J Clin Immunol 2008;28:593-599.

35. Gonzalez-Torres C, Gonzalez-Martinez H, Miliar A, et al.: Effect of malnutrition on the expression of cytokines involved in Th1 cell differentiation. Nutrients 2013;5:579593.

36. Wherry EJ: T cell exhaustion. Nat Immunol 2011;12:492499.

37. D'Souza M, Fontenot AP, Mack DG, et al.: Programmed death 1 expression on HIV-specific CD4+ T cells is driven by viral replication and associated with $\mathrm{T}$ cell dysfunction. J Immunol 2007;179:1979-1987.

38. Sachdeva M, Fischl MA, Pahwa R, Sachdeva N, Pahwa S: Immune exhaustion occurs concomitantly with immune activation and decrease in regulatory $\mathrm{T}$ cells in viremic chronically HIV-1-infected patients. J Acquir Immune Defic Syndr 2010;54:447-454.

39. Vollbrecht $\mathrm{T}$, Brackmann $\mathrm{H}$, Henrich $\mathrm{N}$, et al.: Impact of changes in antigen level on CD38/PD-1 co-expression on HIV-specific CD8 T cells in chronic, untreated HIV-1 infection. J Med Virol 2010;82:358-370.

40. Sauce D, Larsen M, Fastenackels S, et al.: HIV disease progression despite suppression of viral replication is associated with exhaustion of lymphopoiesis. Blood 2011; 117:5142-5151.

41. Cassol E, Malfeld S, Mahasha P, et al.: Persistent microbial translocation and immune activation in HIV-1-infected South Africans receiving combination antiretroviral therapy. J Infect Dis 2010;202:723-733.

42. Grabmeier-Pfistershammer K, Steinberger P, Rieger A, Leitner J, Kohrgruber N: Identification of PD-1 as a unique marker for failing immune reconstitution in HIV-1-infected patients on treatment. J Acquir Immune Defic Syndr 2011; 56:118-124.

43. Hunt PW, Martin JN, Sinclair E, et al.: T cell activation is associated with lower CD4+ T cell gains in human immunodeficiency virus-infected patients with sustained viral suppression during antiretroviral therapy. J Infect Dis 2003; 187:1534-1543.

44. Jiang W, Lederman MM, Hunt P, et al.: Plasma levels of bacterial DNA correlate with immune activation and the magnitude of immune restoration in persons with antiretroviral-treated HIV infection. J Infect Dis 2009;199: 1177-1185.

45. Rajasuriar R, Booth $\mathrm{D}$, Solomon A, et al:: Biological determinants of immune reconstitution in HIV-infected patients receiving antiretroviral therapy: The role of interleukin 7 and interleukin 7 receptor alpha and microbial translocation. J Infect Dis 2010;202:1254-1264.

46. Mori M, Adland E, Paioni P, et al:: Sex Differences in Antiretroviral Therapy Initiation in Pediatric HIV Infection. PLoS One 2015;10:e131591.

47. Muenchhoff M, Goulder PJ: Sex differences in pediatric infectious diseases. J Infect Dis 2014;209 Suppl 3:S120-S126.

$$
\begin{array}{r}
\text { Address correspondence to } \\
\text { Maximilian Muenchhoff } \\
\text { Department of Virology } \\
\text { Max von Pettenkofer-Institut } \\
\text { Ludwig-Maximilians-University Munich } \\
\text { Munich } 80336 \\
\text { Germany }
\end{array}
$$

E-mail: muenchhoff@mvp.uni-muenchen.de 